\title{
Optimization of Packet Length for MIMO systems
}

\author{
Ghassan Alnwaimi ${ }^{1}$, Hatem Boujemaa ${ }^{2}$, Kamran Arshad ${ }^{3}$ \\ ${ }^{1}$ King Abdulaziz University, Kingdom of Saudi Arabia \\ ${ }^{2}$ Sup'Com, COSIM Laboratory, Tunisia \\ ${ }^{3}$ Ajman University, United Arab Emirates
}

\begin{abstract}
In this article, a method to enhance the throughput for Multiple Input Multiple Output (MIMO) systems by optimizing packet length is proposed. Two adaptation algorithms are proposed. In the first algorithm, we use the Average Signal to Noise Ratio (ASNR) to choose the optimal packet length and Modulation and Coding Scheme (MCS) in order to maximize the throughput. In the second algorithm, packet length and MCS are adapted with respect to the Instantaneous received SNR (ISNR). This article concludes that the variable packet length gives up to $1.8 \mathrm{~dB}$ gain with respect to the Fixed Packet Length (FPL).
\end{abstract}

Index Terms : MIMO systems, Optimal packet length, Rayleigh fading channels.

\section{Introduction}

Generally, MIMO systems offer larger throughput than Single Input Single Output (SISO) systems [1-5]. Multiple antennas are used to benefit from transmit/receive diversity and providing enhanced system performance. For instance, in wireless systems, Space Time Coding (STC) is used at the transmitter benefiting from transmit diversity. Accordingly, at the receiver, Space Time Decoding (STD) and Maximum Ratio Combining (MRC) can be used to benefit from spatial diversity and providing higher data rates [1-5].

Channel estimation for MIMO systems has been investigated in the literature e.g. [1]. Power allocation was studied in [2-3] to improve the overall system performance. Scheduling for MIMO systems was considered in [4] and full duplex MIMO was studied in [5-6]. Antenna selection allows to benefit from spatial diversity [7-8]. The antenna with the highest SNR is selected in Selection Combining (SC) to provide higher capacity gains. The theoretical capacity of MIMO systems was derived in [9-10]. A combination of spatial and cooperative diversity was proposed in [11-14]. Relay nodes are used to amplify or decode the source signal. The destination combines signals from the source and other relays. Multihop relaying for MIMO systems was suggested in [13]. The security aspect of MIMO systems was analyzed in [15] in the presence of an eavesdropper. MIMO systems can also be deployed for Underwater Visible Light Communications (UVLC) [16].

In the existing literature [1-16], the packet length is kept fixed. In this paper, we propose methods to enhance the system throughput of MIMO systems by optimizing packet length. The major contributions of the article are as follows : 
- An algorithm is proposed in this article to compute the Optimal Packet Length (OPL) that maximizes the average throughput of the MIMO system.

- An algorithm is proposed to calculate the OPL that maximizes the instantaneous throughput of MIMO system.

- The two adaptation algorithms are also compared in this article. The first one uses the ASNR to optimize packet length and the MCS. The second algorithm uses ISNR to optimize packet length as well as the MCS.

The remainder of this article is organized as follows; Preliminary results on Packet Error Probability (PEP) are given in section 2. Section 3 proposes an algorithm to compute the OPL using average SNR. Section 4 derives the OPL using the instantaneous SNR. Numerical results are provided in section 5 whereas section 6 concludes the paper.

\section{Preliminary Results}

In this section, we derive the expression of the average Packet Error Probability (PEP) for single carrier systems. The PEP can be upper bounded by [17]

$$
\mathrm{PEP} \leq \int_{0}^{w_{0}} f_{\Gamma}(\gamma) d \gamma
$$

where $f_{\Gamma}(\gamma)$ is the Probability Density Function (PDF) of instantaneous SNR $\Gamma$ and $w_{0}$ is a waterfall threshold.

Equation (1) shows that the PEP for $\gamma \leq w_{0}$, can be approximated to 1 . However, the PEP for $\gamma>w_{0}$ can be approximated to 0 [17].

Hence,

$$
\mathrm{PEP} \leq F_{\Gamma}\left(w_{0}\right),
$$

where $F_{\Gamma}(x)$ is the Cumulative Distribution Function (CDF) of the received SNR. We denote $\bar{\Gamma}=\frac{E_{b}}{N_{0}}$ as the average SNR, where $E_{b}$ is the transmitted energy per bit, $N_{0}$ is the noise Power Spectral Density (PSD) and $w_{0}$ is a waterfall threshold written as [17],

$$
w_{0}=\int_{0}^{+\infty} g(\gamma) d \gamma
$$

where $g(\gamma)$ is the PEP for a given instantaneous SNR, $\gamma=\bar{\Gamma}|h|^{2}$ and $h$ is the channel coefficient.

\subsection{Packet Error Probability (PEP) without error correction}

For uncoded transmission and QAM modulation with size $M$, we have

$$
g(\gamma)=1-\left(1-P_{e s}(\gamma)\right)^{\frac{N+n_{d}}{\log _{2}(M)}},
$$

where $N$ is packet length in bits per, $n_{d}$ is the number of CRC (Cyclic Redundancy Check) bits per packet and $P_{e s}$ is the Symbol Error Probability (SEP) given as, [18]

$$
\begin{gathered}
P_{e s}(\gamma) \simeq 2\left(1-\frac{1}{\sqrt{M}}\right) \operatorname{erfc}\left(\sqrt{\frac{\log _{2}(M) 3 \gamma}{(M-1) 2}}\right) \\
\operatorname{erfc}(x) \leq \exp \left(-x^{2}\right)
\end{gathered}
$$


International Journal of Computer Networks \& Communications (IJCNC) Vol.11, No.1, January 2019

Using (5) and (6), we have

$$
P_{e s} \simeq a_{1} e^{-c_{1} \gamma}
$$

where,

$$
\begin{gathered}
a_{1}=2\left(1-\frac{1}{\sqrt{M}}\right), \\
c_{1}=\frac{3 \log _{2}(M)}{2(M-1)}
\end{gathered}
$$

\subsection{PEP with error correction}

If convolutional encoding is used, the PEP is written as

$$
g(\gamma)=1-\left(1-P_{E}(\gamma)\right)^{\frac{N+n_{d}}{\log _{2}(M)}}
$$

where

$$
P_{E}(\gamma) \leq \sum_{d=d_{f}}^{+\infty} a_{d} P_{d}(\gamma)
$$

$d_{f}$ and $a_{d}$ are distance spectral of the convolutional encoder,

$$
P_{d}(\gamma) \simeq 2\left(1-\frac{1}{\sqrt{M}}\right) \operatorname{erfc}\left(\sqrt{\frac{3 R_{c} d \gamma \log _{2}(M)}{2(M-1)}}\right)
$$

where $R_{c}$ is channel encoder rate.

Using (6), we have

$$
P_{E}(\gamma) \simeq a_{2} e^{-c_{2} \gamma}
$$

where

$$
\begin{gathered}
a_{2}=a_{d_{f}} 2\left(1-\frac{1}{\sqrt{M}}\right), \\
c_{2}=\frac{3 R_{c} d_{f} \log _{2}(M)}{2(M-1)} .
\end{gathered}
$$

Hence, we can write $g(\gamma)$ as follow,

$$
g(\gamma) \simeq 1-\left(1-a_{i} e^{-c_{i} \gamma}\right)^{\frac{N+n_{d}}{\log _{2}(M)}},
$$

where $i=1$ for uncoded communications and $i=2$ for coded communications. 


\subsection{Waterfall Threshold}

Using (3) and (16), one can approximate waterfall threshold as follows [19],

$$
w_{0} \simeq k_{1} \ln \left(\frac{N+n_{d}}{\log _{2}(M)}\right)+k_{2}
$$

where,

$$
\begin{gathered}
k_{1}=\frac{1}{c_{i}}, \\
k_{2}=\frac{E+\ln \left(a_{i}\right)}{c_{i}},
\end{gathered}
$$

and $E \simeq 0.577$ is the Euler constant.

\section{OPL using ASNR}

In this section, we derive OPL for MIMO systems. Consider a system with one transmitting and two receiving antennas. It is assumed that Space Time Coding (STC) is used at the transmitter and Space Time Decoding (STD) is used at the receiver. The PEP can be written as follows (see Appendix A for Proof),

$$
\operatorname{PEP}(2)=1-e^{-\frac{w_{0}}{\bar{\Gamma}}}\left[1+\frac{w_{0}}{\bar{\Gamma}}\right]
$$

where $\operatorname{PEP}(2)$ refers to the case of two receiving antennas.

Similarly, when there is one transmitting and three receiving antennas, we show in Appendix $\mathrm{B}$ that the PEP can be written as follows,

$$
\operatorname{PEP}(3)=1-e^{-\frac{w_{0}}{\bar{\Gamma}}}\left[1+\frac{w_{0}}{\bar{\Gamma}}+\frac{1}{2}\left(\frac{w_{0}}{\bar{\Gamma}}\right)^{2}\right]
$$

When there are one transmitting and $n$ receiving antennas, we show in appendix $\mathrm{C}$ that the $\mathrm{PEP}$ is equal to

$$
\operatorname{PEP}(n)=1-e^{-\frac{w_{0}}{\bar{\Gamma}}} \sum_{i=0}^{n-1} \frac{1}{i !}\left(\frac{w_{0}}{\bar{\Gamma}}\right)^{i}
$$

Hence, for the case of $n_{t}$ transmitting and $n_{r}$ receiving antennas, the SNR per bit can be expressed as follows,

$$
\gamma=\frac{E_{b}}{n_{t} N_{0}} \sum_{i=1}^{n_{t}} \sum_{j=1}^{n_{r}} \gamma_{j, i}
$$

where $\gamma_{j, i}$ is the instantaneous SNR between the $i$-th transmitting antenna and $j$-th receiving antenna.

Using the results of Appendix C, the PEP when there are $n_{t}$ transmitting and $n_{r}$ receiving antennas can be written as follows,

$$
\operatorname{PEP}\left(n_{t} n_{r}\right)=1-e^{-\frac{w_{0}}{\bar{\Gamma}}} \sum_{i=0}^{n_{t} n_{r}-1} \frac{1}{i !}\left(\frac{w_{0}}{\bar{\Gamma}}\right)^{i}=1-\Gamma\left(\frac{w_{0}}{\bar{\Gamma}}, n_{t} n_{r}\right)
$$


where

$$
\Gamma(x, n)=\frac{1}{(n-1) !} \int_{x}^{+\infty} t^{n-1} e^{-t} d t .
$$

The average SNR (ASNR) per antenna is

$$
\bar{\Gamma}=\frac{E_{b}}{n_{t} N_{0}}
$$

The average number of attempts of Hybrid Automatic Repeat reQuest (HARQ) protocol is equal to

$$
T_{r}=\sum_{i=1}^{+\infty} \mathrm{PEP}^{i-1}(1-\mathrm{PEP})=\frac{1}{1-\mathrm{PEP}}
$$

Therefore, the system throughput is expressed as follows,

$$
\begin{aligned}
T h r & =\frac{N \log _{2}(M)}{\left(N+n_{d}\right) T_{r}}=\frac{N \log _{2}(M)}{\left(N+n_{d}\right)}(1-\mathrm{PEP}) \\
& =\frac{N \log _{2}(M)}{\left(N+n_{d}\right)} \Gamma\left(\frac{w_{0}}{\bar{\Gamma}}, n_{t} n_{r}\right)
\end{aligned}
$$

The OPL can be obtained using the Gradient algorithm:

$$
N_{i+1}=N_{i}+\mu \frac{\partial \operatorname{Thr}\left(N=N_{i}\right)}{\partial N}
$$

where

$$
\frac{\partial T h r}{\partial N}=\frac{\log _{2}(M) n d}{\left(N+n_{d}\right)^{2}} \Gamma\left(\frac{w_{0}}{\bar{\Gamma}}, n_{t} n_{r}\right)-\frac{\log _{2}(M) N}{\left(N+n_{d}\right)\left(n_{t} n_{r}-1\right) !}\left(\frac{w_{0}}{\bar{\Gamma}}\right)^{n_{t} n_{r}-1} e^{-\frac{w_{0}}{\bar{\Gamma}}} \frac{k_{1}}{\left(N+n_{d}\right) \bar{\Gamma}}
$$

The principle of the OPL using average SNR is shown in Fig. 1.

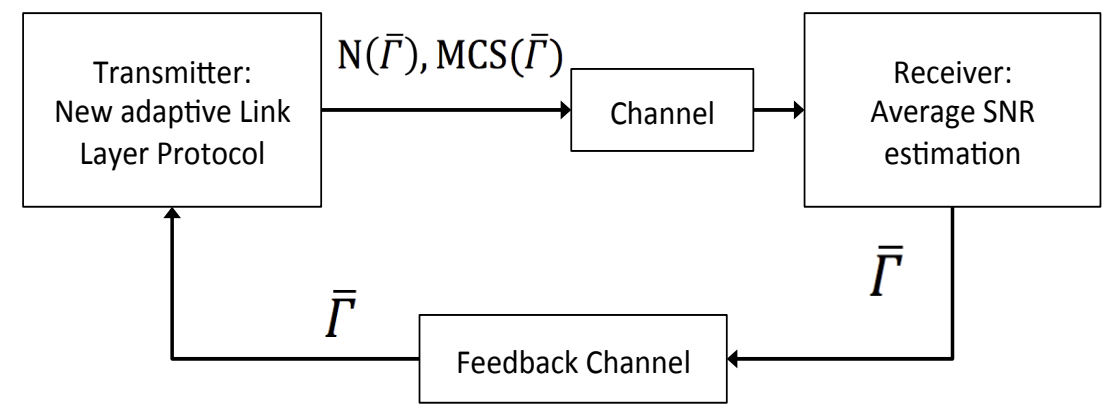

Figure 1: OPL using Average SNR.

\section{OPL using ISNR}

\subsection{Adaptive Packet Length without Channel Coding}

The instantaneous throughput can be expressed as 


$$
\begin{gathered}
\operatorname{Thr}(\gamma)=\frac{N \log _{2}(M)}{N+n_{d}}(1-\operatorname{Pbloc}(\gamma)) \\
=\frac{N \log _{2}(M)}{N+n_{d}}\left(1-\operatorname{Pes}_{M-Q A M}(\gamma)\right)^{\frac{N+n_{d}}{\log _{2}(M)}},
\end{gathered}
$$

where $\operatorname{Pbloc}(\gamma)$ is the packet error probability, $\gamma$ is the instantaneous SNR at the receiver expressed as

$$
\gamma=\frac{E_{b}}{n_{t} N_{0}} \sum_{i=1}^{n_{t}} \sum_{j=1}^{n_{r}} \gamma_{j, i}
$$

$P e s_{M-Q A M}$ is the symbol error probability of M-QAM modulation:

$$
1-\operatorname{Pes}_{M-Q A M}(\gamma)=\left(1-\operatorname{Pes}_{A S K}\right)^{2},
$$

where

$$
\operatorname{Pes}_{A S K}(\gamma)=\left(1-\frac{1}{\sqrt{M}}\right) \operatorname{erfc}\left(\sqrt{\gamma \frac{3 \log _{2}(M)}{2(M-1)}}\right) .
$$

By maximizing the throughput (31), the OPL is given by

$$
N(\gamma)=\frac{n_{d}}{2}\left[\sqrt{1-\frac{2 \log _{2}(M)}{n_{d} \ln \left(1-\operatorname{Pes}_{A S K}(\gamma)\right)}}-1\right]
$$

\subsection{Adaptive Packet Length with Channel Coding}

In this section, a convolutional encoder is employed and similar methodology can be used to derive OPL for other types of channel coding. The throughput can be written as,

$$
\begin{gathered}
T h r_{Q P S K, R c}(\gamma)=\frac{R_{c} 2 N}{\left(N+n_{d}\right)}(1-\operatorname{Pbloc}(\gamma)) \\
=\frac{R_{c} 2 N}{N+n_{d}}\left(1-P_{E}(\gamma)\right)^{\frac{N+n_{d}}{2}}
\end{gathered}
$$

where

$$
P_{E}(\gamma)<\min \left(1, \sum_{d=d_{f}}^{+\infty} a_{d} \mathrm{Q}\left(\sqrt{2 d R_{c} \gamma}\right)\right)
$$

When convolutional coding is employed, the OPL can be written as follows

$$
N(\gamma)=\frac{n_{d}}{2}\left[\sqrt{1-\frac{8}{n_{d} \ln \left(1-P_{E}(\gamma)\right)}}-1\right]
$$

The expression of OPL is obtained by maximizing the throughput given in (36). 


\subsection{OPL and optimal MCS}

From Fig. 2, it can be deduced easily that different threshold should be used to select different MCS:

- Case $1:$ for $\left(\gamma \leq F_{1}=6.3 d B\right) \rightarrow$ use a coded QPSK modulation with rate $R_{c}=1 / 2$..

- Case 2 : for $\left(F_{1}<\gamma \leq F_{2}=10 d B\right) \rightarrow$ use uncoded QPSK.

- Case 3 : for $\left(F_{2}<\gamma \leq F_{3}=15.2 d B\right) \rightarrow$ use uncoded 16-QAM.

- Case $4:$ for $\left(F_{3}<\gamma\right) \rightarrow$ use uncoded 64 QAM.

Packet length is given in (38) or (35).

Thresholds $F_{i}$ are defined in Figure 2.

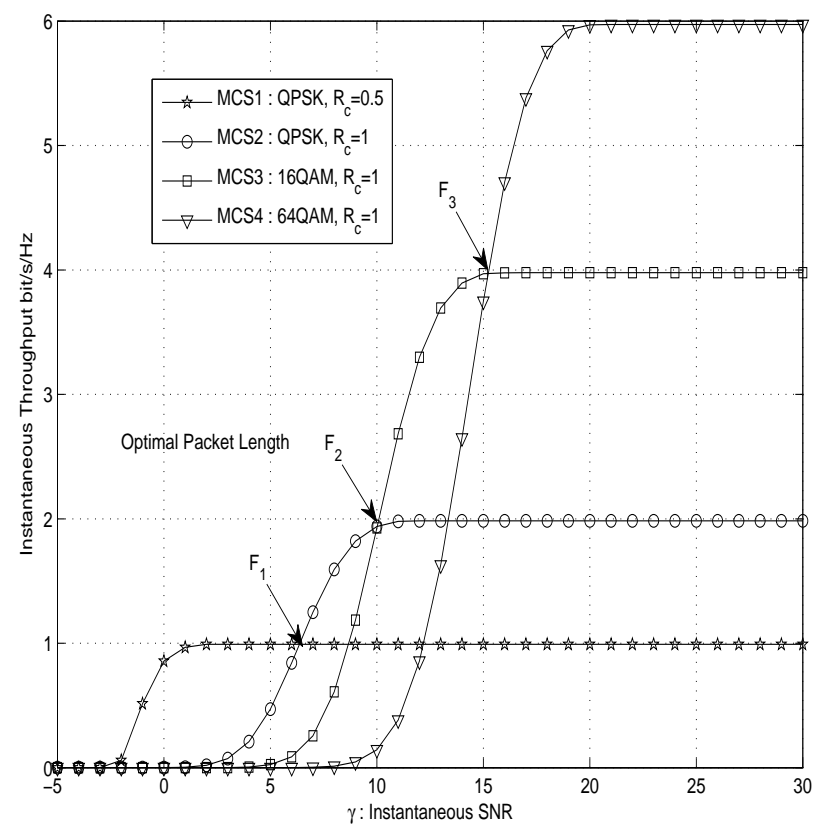

Figure 2: Thresholds to select MCS using instantaneous SNR : OPL.

When the MCS is adapted for $N=590$ and $n_{d}=10$, Fig. 3 shows that we have to use other thresholds : $Z_{1}=5.4 \mathrm{~dB}, Z_{2}=9 \mathrm{~dB}$ and $Z_{3}=13.8 \mathrm{~dB}$. 


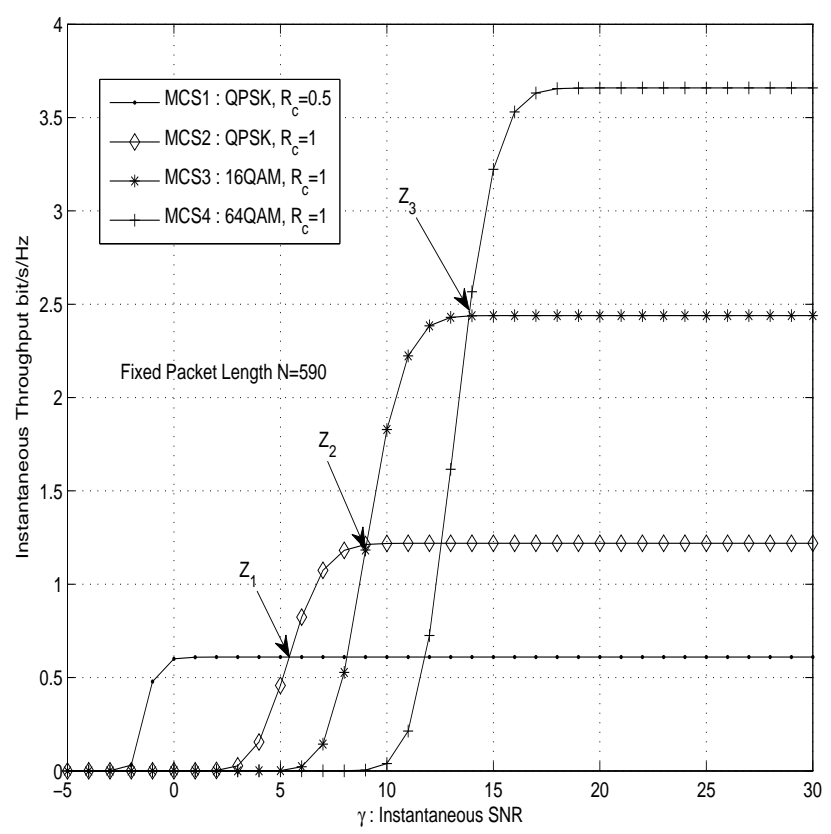

Figure 3: Thresholds to select MCS using instantaneous SNR : FPL.

Fig. 4 shows the principle of the proposed packet length and MCS adaptation using ISNR. The ISNR is used to select the optimal MCS and OPL as shown in (35) and (38).

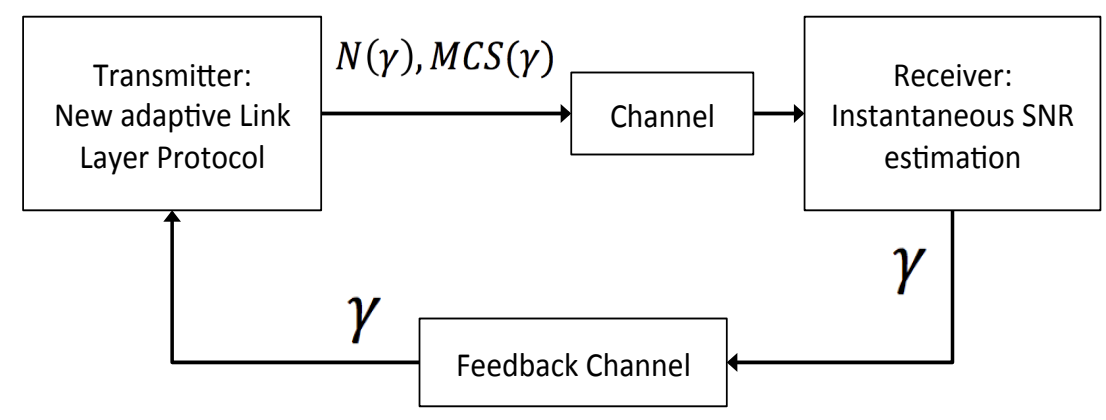

Figure 4: OPL and Adaptive MCS using Instantaneous SNR.

For a given average $\mathrm{SNR}, \bar{\Gamma}=\frac{E_{b}}{n_{t} N_{0}}$, the average throughput is written as 


$$
\begin{aligned}
\operatorname{Thr}(\bar{\Gamma})= & \int_{0}^{F_{1}} T h r_{Q P S K, R c}(\gamma) p(\gamma) d \gamma \\
& +\int_{F_{1}}^{F_{2}} \operatorname{Thr}_{Q P S K}(\gamma) p(\gamma) d \gamma \\
& +\int_{F_{2}}^{F_{3}} T h r_{16-Q A M}(\gamma) p(\gamma) d \gamma \\
& +\int_{F_{3}}^{+\infty} \operatorname{Thr}_{64-Q A M}(\gamma) p(\gamma) d \gamma
\end{aligned}
$$

where $p(\gamma)$ is the PDF of the instantaneous SNR $\gamma$. For Rayleigh fading channel, the SNR in (23) follows a central chi-square distribution with $2 n_{t} n_{r}$ degrees of freedom given by

$$
p(\gamma)=\frac{1}{\sigma^{n_{t} n_{r}} 2^{n_{t} n_{r} / 2}\left(n_{t} n_{r} / 2-1\right) !} \gamma^{n_{t} n_{r} / 2-1} e^{-\frac{\gamma}{2 \sigma^{2}}}, \gamma \geq 0,
$$

where $\sigma^{2}=\frac{E_{b}}{2 N_{0} n_{t}}$.

Equation (39) gives the throughput for OPL when MCS is also adapted to ISNR. The expressions of throughput given in (31) and (35) must be used with packet length $N(\gamma)$ as given in (35) and (38) that depends on instantaneous SNR $\gamma$. When MCS is adapted for a FPL $N(\gamma)=N$, we have to use (39) with different thresholds $Z_{1}, Z_{2}$ and $Z_{3}$.

\section{Theoretical and Simulation Results}

Simulation results were obtained using MATLAB as the simulation environment.

Simulation results were performed by measuring the Packet Error Rate (PER) to deduce the throughput. The packet error rate is the number of erroneous packets/number of transmitted packets. We made simulation until 1000 packets are erroneously received.

Fig. 5 shows the average throughput of MIMO systems with respect to packet length for QPSK modulation and for average SNR per bit of $E_{b} / N_{0}=6 \mathrm{~dB}$. We studied SISO systems $n_{t}=n_{r}=1$ as well as MIMO for $\left(n_{t}, n_{r}\right)=(1,2),\left(n_{t}, n_{r}\right)=(2,2),\left(n_{t}, n_{r}\right)=(2,3),\left(n_{t}, n_{r}\right)=$ $(3,3)$. Space Time Coding (STC) is used at the transmitter and Space Time Decoding (STD) is used at the receiver in our simulation. It is concluded in this article that the packet length optimization allows increasing data rates for MIMO systems. Furthermore, the packet length is also dependent on the number of transmitting and receiving antenna; packet length should be increased as the number of transmitting and receiving antennas increase. 


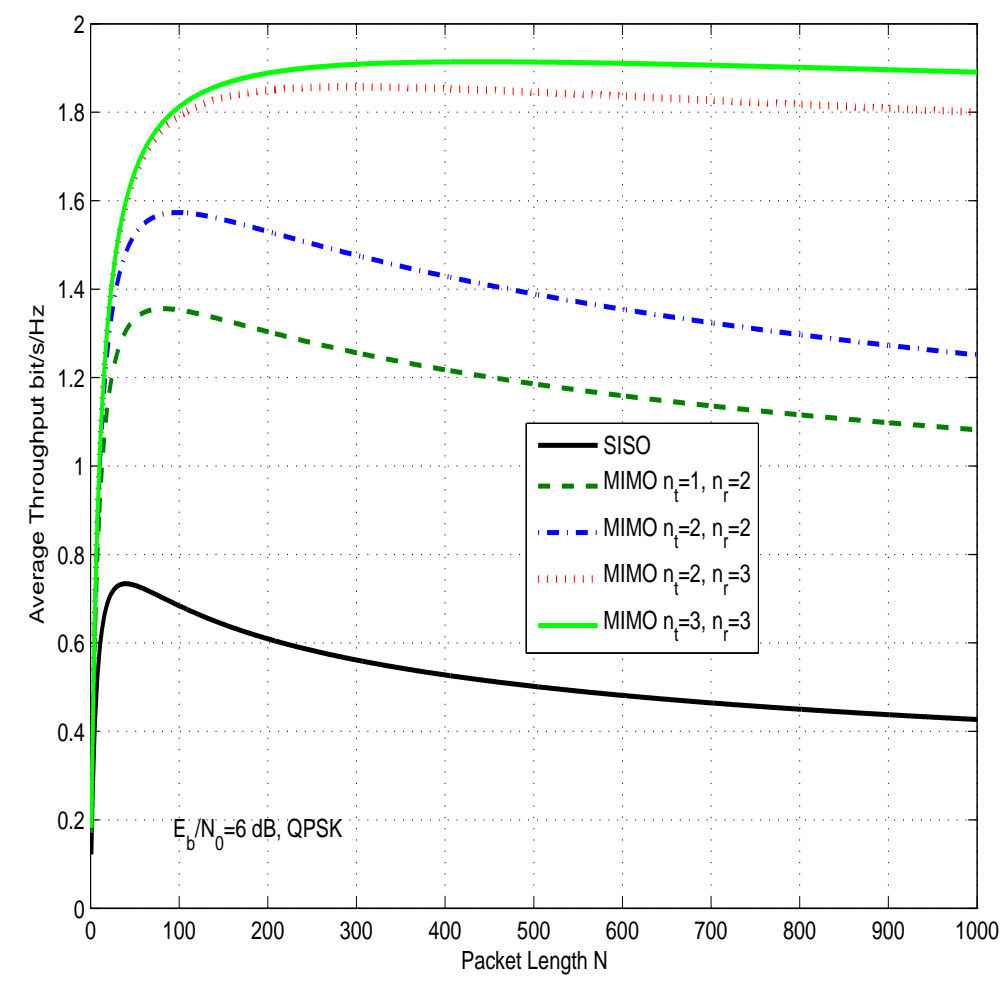

Figure 5: Throughput of MIMO systems with respect to packet length.

Fig. 6 shows the OPL for QPSK modulation and different MIMO systems. We notice that packet length increases with Average SNR (ASNR). Packet length must be increased as the number of transmitting and receiving antennas increase. 


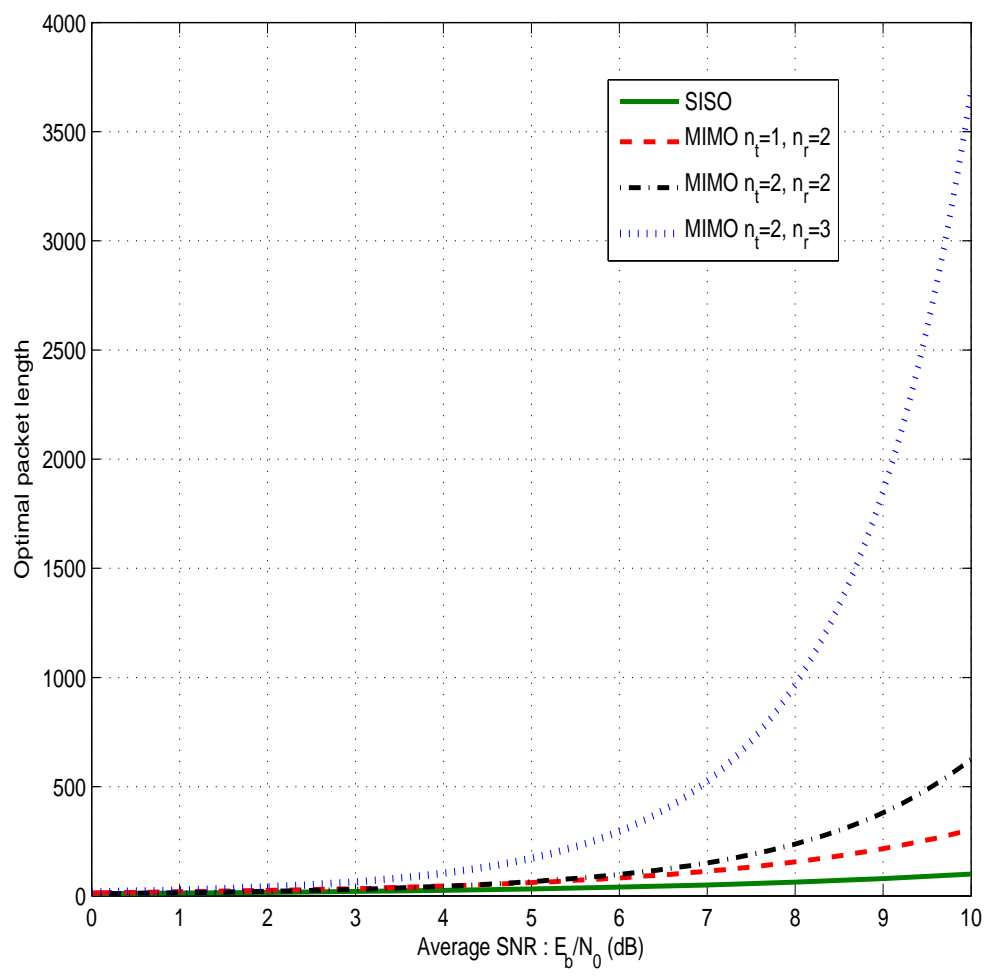

Figure 6: OPL for MIMO systems.

Fig. 7 shows the throughput of MIMO systems using OPL for $n_{t}=2$ transmitting and $n_{r}=2$ receiving antennas. Different Modulation and Coding Schemes (MCS) are studied : QPSK with convolutional channel coding and rate $R_{c}=0.5$, generator polynomials $(133,171)$, memory 6 and free distance 10. uncoded QPSK, 16 QAM and 64 QAM. From Fig. 7, we can note that the following strategy should be employed :

- Case $1:$ for $\left(\bar{\Gamma} \leq S_{1}=2.3 d B\right) \rightarrow$ We should use a coded QPSK modulation with rate $R_{c}=0.5$.

- Case 2 : for $\left(S_{1}<\bar{\Gamma} \leq S_{2}=6.8 d B\right) \rightarrow$ We should use an uncoded QPSK modulation.

- Case 3 : for $\left(S_{2}<\bar{\Gamma} \leq S_{3}=12.6 d B\right) \rightarrow$ We should use an uncoded 16 QAM modulation.

- Case $4:$ for $\left(S_{3}<\bar{\Gamma}\right) \rightarrow$ We should use an uncoded 64 QAM modulation.

Thresholds $S_{i}$ are defined in Fig. 7 . 


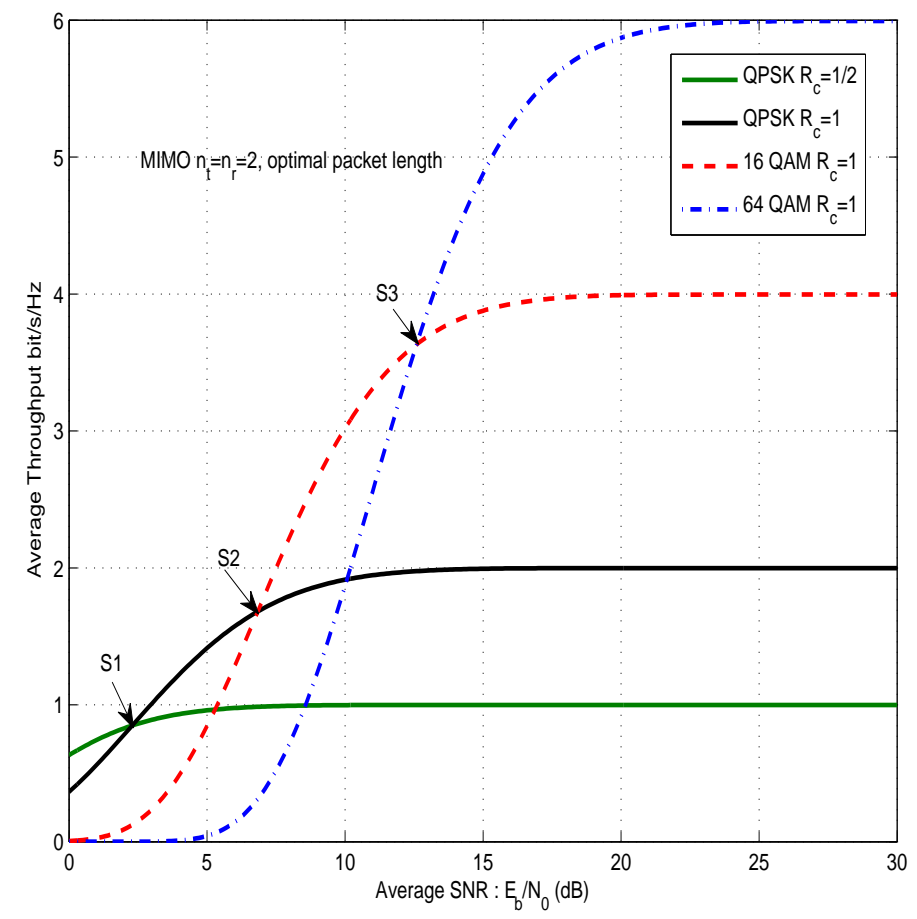

Figure 7: Throughput of MIMO systems for OPL and different modulations.

Fig. 8 shows the throughput of MIMO systems using FPL $\mathrm{N}=590$ for $n_{t}=2$ transmitting and $n_{r}=2$ receiving antennas. Different Modulation and Coding Schemes (MCS) are studied : QPSK with convolutional channel coding and rate $R_{c}=0.5$, generator polynomials $(133,171)$, memory 6 and free distance 10. uncoded QPSK, 16 QAM and 64 QAM. From Fig. 8, it can be noted that the following strategy should be employed :

- Case 1 : for $\left(\bar{\Gamma} \leq D_{1}=4.7 d B\right) \rightarrow$ We should use a coded QPSK modulation with rate $R_{c}=0.5$.

- Case 2 : for $\left(D_{1}<\bar{\Gamma} \leq D_{2}=8.9 d B\right) \rightarrow$ We should use an uncoded QPSK modulation.

- Case 3 : for $\left(D_{2}<\bar{\Gamma} \leq D_{3}=14.3 d B\right) \rightarrow$ We should use an uncoded 16 QAM modulation.

- Case $4:$ for $\left(D_{3}<\bar{\Gamma}\right) \rightarrow$ We should use an uncoded 64 QAM modulation.

Thresholds $D_{i}$ are defined in Fig. 8. 


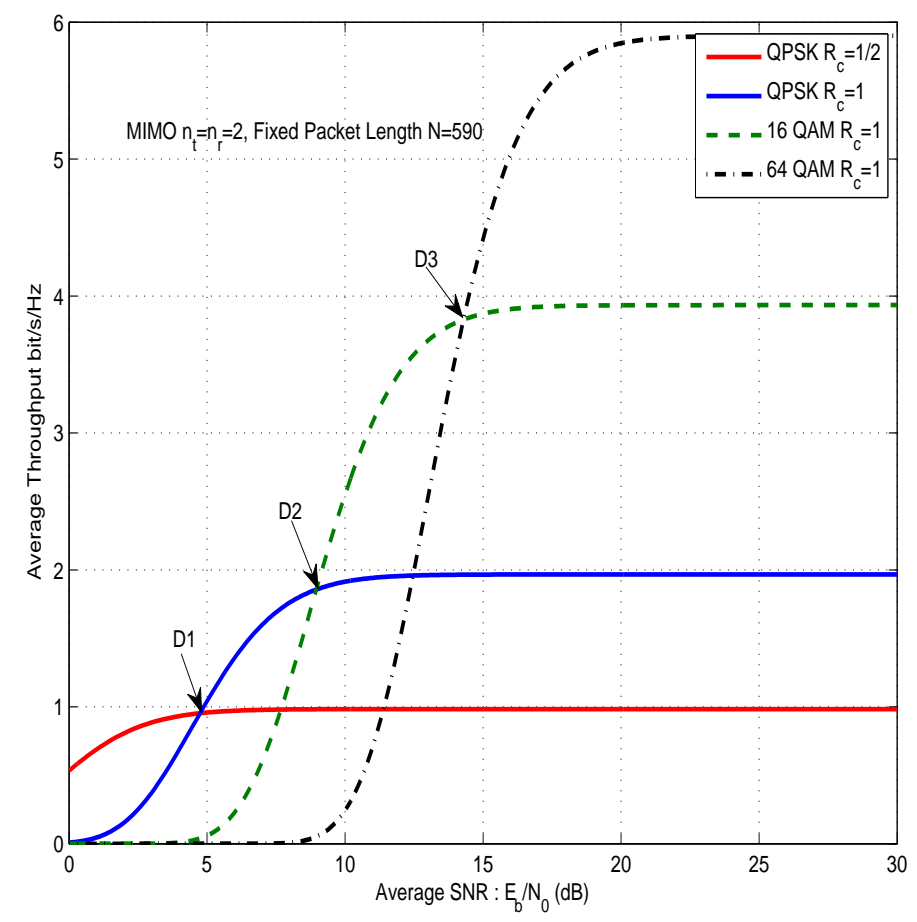

Figure 8: Throughput of MIMO systems for FPL and different modulations.

Fig. 9 shows the average throughput of MIMO systems with two transmitting and two receiving antennas. In Fig. 9, we compare the two approaches: the first one consists to optimize the MCS using average SNR with FPL, $N=590$. The second approach is to optimize the MCS and packet length using the average SNR. The proposed strategy offers $2 \mathrm{~dB}$ gains with respect to FPL for a throughput of $4 \mathrm{bit} / \mathrm{s} / \mathrm{Hz}$. 


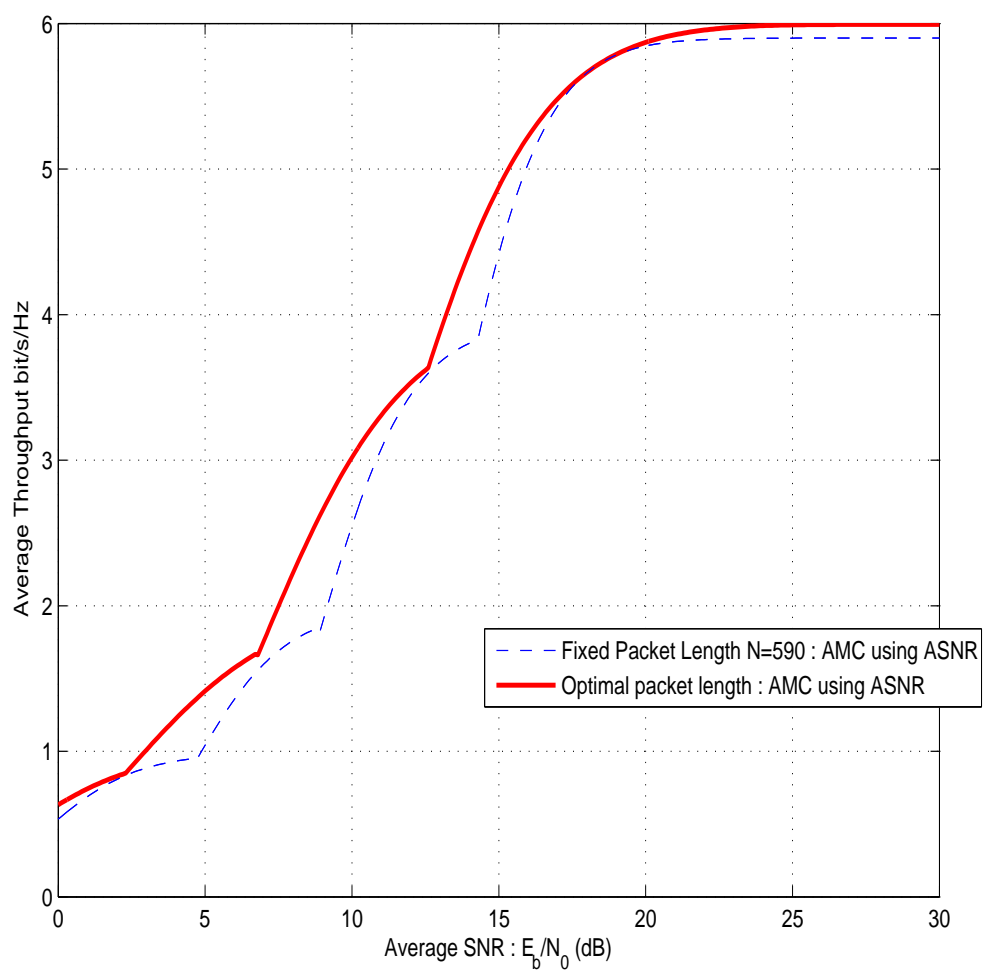

Figure 9: Throughput of MIMO systems with AMC using ASNR : fixed versus OPL.

Fig. 10 shows the throughput for two transmitting and two receiving antennas $n_{t}=n_{r}=2$ : We studied:

- Optimal packet length (OPL) and MCS using ASNR

- OPL and MCS using ISNR

- Optimal MCS using ASNR with Fixed Packet Length (FPL).

- Optimal MCS using ISNR with FPL.

First, we noticed that the best strategy is to use the OPL and MCS using ISNR as explained in section 4 . The proposed protocol offers $1.8 \mathrm{~dB}$ gain with respect to optimal MCS and FPL as considered in [1-16]. 


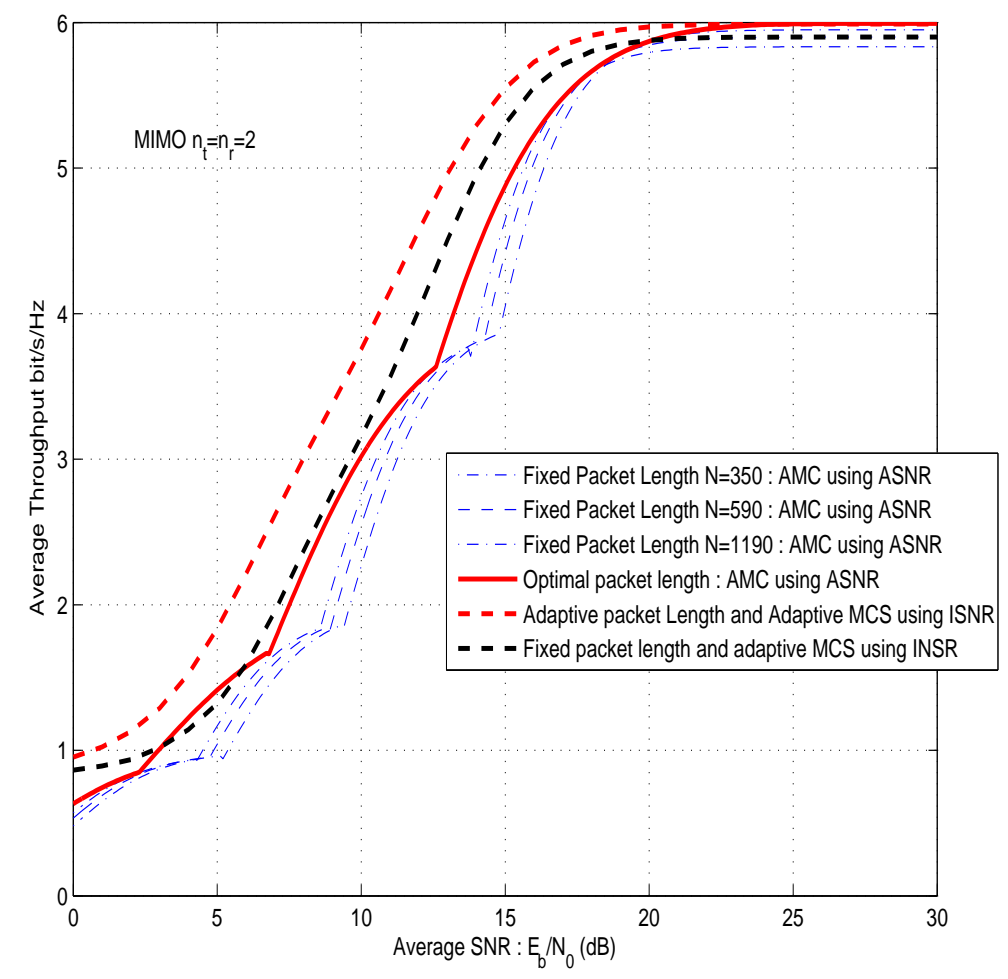

Figure 10: Throughput of MIMO systems with AMC using ASNR or ISNR : fixed versus OPL.

\section{Conclusion}

In this article, we have proposed strategies to enhance the throughput for MIMO systems by optimizing packet length. Two adaptation algorithms are proposed and evaluated in this article. In the first one, for each received ASNR, the OPL and optimal Modulation and Coding Scheme (MCS) can be selected. Therefore, packet length adaptation is performed with respect to ASNR. In the second approach, the adaptation is made with respect to ISNR. The proposed OPL allows $1.8 \mathrm{~dB}$ gains with respect to FPL.

\section{Appendix A :}

When there are two receiving antennas, the PEP can be upper bounded by

$$
\operatorname{PEP}(2) \leq \iint_{x_{1}+x_{2} \leq w_{0}} f_{\Gamma}\left(x_{1}\right) f_{\Gamma}\left(x_{2}\right) d x_{1} d x_{2}
$$

where $f_{\Gamma}(x)$ is the PDF of SNR $\Gamma$.

For Rayleigh fading channels, the SNR follows an exponential distribution so that we can write

$$
\operatorname{PEP}(2) \leq \int_{0}^{w_{0}} \frac{e^{-\frac{x_{1}}{\bar{\Gamma}}}}{\bar{\Gamma}} \int_{0}^{w_{0}-x_{1}} \frac{e^{-\frac{x_{2}}{\bar{\Gamma}}}}{\bar{\Gamma}} d x_{2} d x_{1}=1-e^{-\frac{w_{0}}{\bar{\Gamma}}}\left[1+\frac{w_{0}}{\bar{\Gamma}}\right]
$$

\section{Appendix B :}

When there are three receiving antennas, the PEP can be upper bounded by

$$
\operatorname{PEP}(3) \leq \iiint_{x_{1}+x_{2}+x_{3} \leq w_{0}} f_{\Gamma}\left(x_{1}\right) f_{\Gamma}\left(x_{2}\right) f_{\Gamma}\left(x_{3}\right) d x_{1} d x_{2} d x_{3}
$$


For Rayleigh fading channels, we can write

$$
\operatorname{PEP}(3) \leq \int_{0}^{w_{0}} \frac{e^{-\frac{x_{1}}{\bar{\Gamma}}}}{\bar{\Gamma}} \int_{0}^{w_{0}-x_{1}} \frac{e^{-\frac{x_{2}}{\bar{\Gamma}}}}{\bar{\Gamma}} \int_{0}^{w_{0}-x_{1}-x_{2}} \frac{e^{-\frac{x_{3}}{\bar{\Gamma}}}}{\bar{\Gamma}} d x_{3} d x_{2} d x_{1}
$$

We deduce that

$$
\operatorname{PEP}(3) \leq \int_{0}^{w_{0}} \frac{e^{-\frac{x_{1}}{\bar{\Gamma}}}}{\bar{\Gamma}} \int_{0}^{w_{0}-x_{1}} \frac{e^{-\frac{x_{2}}{\bar{\Gamma}}}}{\bar{\Gamma}}\left[1-e^{\frac{x_{1}+x_{2}-w_{0}}{\bar{\Gamma}}}\right] d x_{2} d x_{1}
$$

Therefore, we have

$$
\operatorname{PEP}(3) \leq \int_{0}^{w_{0}} \frac{e^{-\frac{x_{1}}{\bar{\Gamma}}}}{\bar{\Gamma}}\left[1-e^{-\frac{x_{1}-w_{0}}{\bar{\Gamma}}}+\frac{x_{1}-w_{0}}{\bar{\Gamma}} e^{\frac{x_{1}-w_{0}}{\bar{\Gamma}}}\right] d x_{1}
$$

Finally, we have

$$
\operatorname{PEP}(3) \leq 1-e^{-\frac{w_{0}}{\bar{\Gamma}}}\left[1+\frac{w_{0}}{\bar{\Gamma}}+\frac{w_{0}^{2}}{2 \bar{\Gamma}^{2}}\right]
$$

\section{Appendix C:}

When there are $n$ receiving antennas, the PEP can be written as

$$
\operatorname{PEP}(n) \leq \iint \ldots \int_{x_{1}+\ldots+x_{n} \leq w_{0}} f_{\Gamma}\left(x_{1}\right) f_{\Gamma}\left(x_{2}\right) \ldots f_{\Gamma}\left(x_{n}\right) d x_{1} d x_{2} \ldots d x_{n},
$$

We deduce that

$$
\operatorname{PEP}(n) \leq \int_{0}^{w_{0}} \frac{e^{-\frac{x_{1}}{\bar{\Gamma}}}}{\bar{\Gamma}} \int_{0}^{w_{0}-x_{1}} \frac{e^{-\frac{x_{2}}{\bar{\Gamma}}}}{\bar{\Gamma}} \ldots \int_{0}^{w_{0}-\sum_{i=1}^{n-1} x_{i}} \frac{e^{-\frac{x_{n}}{\bar{\Gamma}}}}{\bar{\Gamma}} d x_{n} d x_{n-1} \ldots d x_{1}
$$

and

$$
\operatorname{PEP}(n) \leq \int_{0}^{w_{0}} \frac{e^{-\frac{x_{1}}{\bar{\Gamma}}}}{\bar{\Gamma}} \int_{0}^{w_{0}-x_{1}} \frac{e^{-\frac{x_{2}}{\bar{\Gamma}}}}{\bar{\Gamma}} \ldots \int_{0}^{w_{0}-\sum_{i=1}^{n-2} x_{i}}\left[1-e^{\frac{\sum_{i=1}^{n-1} x_{i}-w_{0}}{\bar{\Gamma}}}\right] \frac{e^{-\frac{x_{n-1}}{\bar{\Gamma}}}}{\bar{\Gamma}} d x_{n-1} \ldots d x_{1}
$$

We compute the last integral to obtain

$$
\begin{gathered}
\operatorname{PEP}(n) \leq \int_{0}^{w_{0}} \frac{e^{-\frac{x_{1}}{\bar{\Gamma}}}}{\bar{\Gamma}} \int_{0}^{w_{0}-x_{1}} \frac{e^{-\frac{x_{2}}{\bar{\Gamma}}}}{\bar{\Gamma}} \ldots \\
\int_{0}^{w_{0}-\sum_{i=1}^{n-3} x_{i}}\left[1-e^{\frac{\sum_{i=1}^{n-2} x_{i}-w_{0}}{\bar{\Gamma}}}\left(1-\frac{\sum_{i=1}^{n-2} x_{i}-w_{0}}{\bar{\Gamma}}\right)\right] \frac{e^{-\frac{x_{n-2}}{\bar{\Gamma}}}}{\bar{\Gamma}} d x_{n-2} \ldots d x_{1}
\end{gathered}
$$

The first two terms of (51) are computed similarly to (50) to have

$$
\begin{gathered}
\operatorname{PEP}(n) \leq \int_{0}^{w_{0}} \frac{e^{-\frac{x_{1}}{\bar{\Gamma}}}}{\bar{\Gamma}} \int_{0}^{w_{0}-x_{1}} \frac{e^{-\frac{x_{2}}{\bar{\Gamma}}}}{\bar{\Gamma}} \ldots \\
\int_{0}^{w_{0}-\sum_{i=1}^{n-4} x_{i}}\left[1-e^{\frac{\sum_{i=1}^{n-3} x_{i}-w_{0}}{\bar{\Gamma}}}\left(1-\frac{\sum_{i=1}^{n-3} x_{i}-w_{0}}{\bar{\Gamma}}+\frac{\left(\sum_{i=1}^{n-3} x_{i}-w_{0}\right)^{2}}{2 \bar{\Gamma}^{2}}\right)\right] \frac{e^{-\frac{x_{n-3}}{\bar{\Gamma}}}}{\bar{\Gamma}} d x_{n-3} \ldots . . . d x_{1}
\end{gathered}
$$


The last integral is as follows

$\operatorname{PEP}(n) \leq \int_{0}^{w_{0}} \frac{e^{-\frac{x_{1}}{\bar{\Gamma}}}}{\bar{\Gamma}}\left[1-e^{\frac{x_{1}-w_{0}}{\bar{\Gamma}}}\left(1-\frac{x_{1}-w_{0}}{\bar{\Gamma}}+\frac{\left(x_{1}-w_{0}\right)^{2}}{2 \bar{\Gamma}^{2}}+\ldots+(-1)^{n} \frac{\left(x_{1}-w_{0}\right)^{n}}{n ! \bar{\Gamma}^{n}}\right)\right] d x_{1}$

We finally obtain

$$
\operatorname{PEP}(n) \leq 1-e^{\frac{-w_{0}}{\bar{\Gamma}}} \sum_{i=0}^{n} \frac{1}{i !}\left(\frac{w_{0}}{\bar{\Gamma}}\right)^{i}
$$

\section{References}

[1] Pallaviram Sure; Narendra Babu C; Chandra Mohan Bhuma, "Large random matrix-based channel estimation for massive MIMO-OFDM uplink", IET Communications, Year: 2018, Volume: 12, Issue: 9 Pages: 1035 - 1041.

[2] Chung Duc Ho; Hien Quoc Ngo; Michail Matthaiou; Long D. Nguyen, "Power Allocation for Multi-Way Massive MIMO Relaying", IEEE Transactions on Communications, Year: 2018, ( Early Access ), Pages: 1 - 1.

[3] Guili Zhou; Youming Li; Yu-Cheng He; Xiaoli Wang; Mingchen Yu, "Artificial fish swarm based power allocation algorithm for MIMO-OFDM relay underwater acoustic communication", IET Communications, Volume: 12, Issue: 9 Pages: 1079 - 1085, 2018.

[4] Narayan Prasad; Xiao Feng Qi, "Downlink multi-user MIMO scheduling with performance guarantees", 2018 16th International Symposium on Modeling and Optimization in Mobile, Ad Hoc, and Wireless Networks (WiOpt), Pages: 1 - 8, 2018.

[5] Jawad Mirza; Gan Zheng; Kai-Kit Wong; Sangarapillai Lambotharan; Lajos Hanzo, "On the Performance of Multiuser MIMO Systems Relying on Full-Duplex CSI Acquisition", IEEE Transactions on Communications, Year: 2018.

[6] Parag Aggarwal; Vivek Ashok Bohara, "Analytical Characterization of Dual-Band MultiUser MIMO-OFDM System with Nonlinear Transmitter Constraints", IEEE Transactions on Communications, Year: 2018.

[7] Muhammad Hanif; Hong-Chuan Yang; Gary Boudreau; Edward Sich; Hossein Seyedmehdi, "Antenna subset selection for massive MIMO systems: A trace-based sequential approach for sum rate maximization", Journal of Communications and Networks, Volume: 20, Issue: 2 Pages: 144 - 155, 2018.

[8] Harun Siljak; Irene Macaluso; Nicola Marchetti, "Distributing Complexity: A New Approach to Antenna Selection for Distributed Massive MIMO", IEEE Wireless Communications Letters, 2018.

[9] Walid Al-Hussaibi; Falah H. Ali, "A Closed-Form Approximation of Correlated Multiuser MIMO Ergodic Capacity with Antenna Selection and Imperfect Channel Estimation", IEEE Transactions on Vehicular Technology, Year: 2018, ( Early Access ). 
[10] Nir Shlezinger; Roee Shaked; Ron Dabora, "On the Capacity of MIMO Broadband Power Line Communications Channels", IEEE Transactions on Communications, Year: 2018, ( Early Access ), Pages: $1-1$.

[11] Chun-Tao Lin; Ronald Y. Chang; Fan-Shuo Tseng, "Source and Relay Precoding for FullDuplex MIMO Relaying with a SWIPT-Enabled Destination", IEEE Communications Letters, Year: 2018, ( Early Access ), Pages: $1-1$.

[12] Cheol Jeong; MinChul Ju; Sung Ho Chae, "MIMO Decode-and-Forward Relay Systems With an Energy-Constrained Antenna-Switching Relay", IEEE Communications Letters, Year: 2017, Volume: 21, Issue: 8, Pages: 1851 - 1854.

[13] Ishtiaq Ahmad; Khoa D. Nguyen; Andre Pollok; Nick Letzepis, "Multi-hopping loss in MIMO decode-and-forward cooperative relaying", 2016 IEEE 27th Annual International Symposium on Personal, Indoor, and Mobile Radio Communications (PIMRC), Year: 2016 Pages: $1-6$

[14] Zhengzheng Xiang; Meixia Tao; Jianhua Mo; Xiaodong Wang, "Degrees of Freedom for MIMO Two-Way X Relay Channel", IEEE Transactions on Signal Processing, Year: 2013, Volume: 61, Issue: 7, Pages: $1711-1720$.

[15] Santosh Timilsina; Gayan Amarasuriya Aruma Baduge; Rafael F. Schaefer, "Secure Communication in Spectrum-Sharing Massive MIMO Systems with Active Eavesdropping", IEEE Transactions on Cognitive Communications and Networking, Year: 2018, ( Early Access ) Pages: $1-1$.

[16] Mohammad Vahid Jamali; Pooya Nabavi; Jawad A. Salehi, "MIMO Underwater Visible Light Communications: Comprehensive Channel Study, Performance Analysis, and Multiple-Symbol Detection", IEEE Transactions on Vehicular Technology, Year: 2018, ( Early Access )

[17] Y. Xi, A. Burr, J. B. Wei, D. Grace, "A general upper bound to evaluate packet error rate over quasi-static fading channels", IEEE Trans. Wireless Communications, vol. 10, nO 5, pp 1373-1377, May 2011.

[18] J. G. Proakis, Digital Communications, Third edition, Mc Graw Hill, 1995.

[19] S. Y. Liu, X. Wu, Y. Xi, J. Wei, "On the throughput and OPL of an uncoded ARQ system over slow Rayleigh fading channels", IEEE Communication Letters, Vol. 16, no 8, pp. 1173-1175, August 2012. 